\title{
Neumonitis de hipersensibilidad asociada con exposición a ácido oxálico: partiendo de un caso
}

\author{
Hypersensitivity pneumonitis associated with \\ exposure to oxalic acid: based on a case
}

Lucy Maritza Pérez Lugo, MD; ${ }^{1}$ Karen Vivanco Payares, MD; ${ }^{1}$ Jennifer lobelo Angulo, MD; ${ }^{1}$ Katya Velásquez, MD; ${ }^{1}$ Lourdes Varela Prieto, Micb. ${ }^{2}$ ERnesto Santiago, MD. ${ }^{3}$

\section{Resumen}

INTRODUCCIÓN: la neumonitis de hipersensibilidad (NH; alveolitis alérgica extrínseca) es una enfermedad inmunológica pulmonar causada por una respuesta inmunitaria exagerada a la inhalación de partículas químicas u orgánicas que afectan los alvéolos, bronquiolos terminales e intersticio alveolar. No existen casos reportados de asociación acido oxáliconeumonitis de hipersensibilidad; además, el infradiagnóstico contribuye al mal pronóstico de la enfermedad, lo que motivó a la presentación del caso.

DESCRIPCIÓN DEL CASO: se trata de una paciente de 35 años de edad con cuadro de disnea y tos seca crónica después de la exposición no laboral a ácido oxálico, pruebas de función pulmonar con patrón restrictivo, tomografía axial computarizada de alta resolución (TACAR) de tórax con patrón sugestivo de NH y biopsia pulmonar de un granuloma tipo colesterol. Se inició el tratamiento sin respuesta, se solicitó trasplante de pulmón actualmente en lista de espera.

DISCUSIÓN: la paciente cumplió criterios para NH sugeridos por Semin y colaboradores (2012), una vez se retiró de noxa no hubo mejoría de los síntomas, así como tampoco presentó mejoría con el uso de corticosteroides, se considera que el diagnóstico y tratamiento tardíos son elementos que modificaron la respuesta al mismo y finalmente alteraron el pronóstico. El trasplante mejora la supervivencia de los pacientes a corto y mediano plazo.

Palabras clave: neumonitis de hipersensibilidad, disnea crónica, ácido oxálico.

\begin{abstract}
INTRODUCTION: Hypersensitivity pneumonitis (HSP; extrinsic allergic alveolitis) is an immunological pulmonary disease caused by an exaggerated immune response to chemical or organic particles inhalation affecting alveoli, terminal bronchioles and alveolar interstitium. There are no reported cases associating oxalic acid-hypersensitivity pneumonitis. In addition, the infra-diagnosis contributes to the poor disease prognosis, which is the reason to present the case.
\end{abstract}

CASE DESCRIPTION: A 35-year-old patient with dyspnea and chronic dry cough after non-occupational exposure to oxalic acid, pulmonary function tests with restrictive pattern, chest high-resolution computed tomography (HRCT) suggestive of HSP and lung biopsy of a granuloma type cholesterol. Treatment is initiated without response, lung transplant requested currently is on waiting list.
${ }^{1}$ Residente de Medicina Interna, Universidad Libre seccional Barranquilla. Barranquilla, Colombia.

${ }^{2}$ Microbióloga, Magíster en Administración y Supervisión Educativa, asesora metodológica, docente asociada a la Universidad Libre Seccional Barranqui1la. Barranquilla, Colombia.

${ }^{3}$ Médico Internista, Neumólogo. Servicio de Neumología, Clínica General del Norte. Docente asociado a la Universidad Libre Seccional Barranquilla. Barranquilla, Colombia. Autor de correspondencia Lucy Maritza Pérez. Correo electrónico: lucyperezlugo@outlook.com.

Recibido: 11/4/2019. Aceptado: 25/5/2019. 
DISCUSSION: The patient met criteria for HSP suggested by Semin et al. (2012), once noxa retired there was no symptoms improvement, as well as the one use of corticoids, the diagnosis and treatment are considered late, elements which modified the response to it and finally altered the prognosis, the transplant improves the survival of patients in the short and medium term.

Keywords: Pneumonitis of hypersensitivity, chronic dyspnea, oxalic acid.

\section{Introducción}

La neumonitis de hipersensibilidad (NH; alveolitis alérgica extrínseca) se define como una enfermedad inmunológica pulmonar causada por una respuesta inmunitaria exagerada a la inhalación de partículas químicas u orgánicas que afecta los alvéolos, bronquiolos terminales e intersticio alveolar (1). La característica primordial es el predominio inflamatorio linfocítico y granulomatoso desproporcionado en el parénquima pulmonar por la inhalación repetida de antígenos en forma de aerosol, en un individuo previamente sensibilizado $(1,2)$. Según su evolución, puede ser aguda o crónica; esta última puede avanzar a fibrosis pulmonar, que constituye una enfermedad incapacitante (3).

Sus primeras descripciones ocurrieron en 1962 cuando se le acuñó el término pulmón granjero por Pepys y colaboradores (4), y fueron ellos quienes realizaron la primera asociación entre las precipitinas del suero de heno y los extractos de moho con la neumonitis de hipersensibilidad (4); desde ese momento, múltiples agentes causales se han descrito, tanto orgánicos (antígenos fúngicos, protozoales, bacterianos y animales) como inorgánicos (químicos de bajo peso molecular y metales) (5).

Su prevalencia es de 0,9 casos por 100000 habitantes (6) y constituye el $1,5 \%$ a $12 \%$ de todas las enfermedades pulmonares intersticiales (7); dicha prevalencia baja explica la tasa alta de subdiagnóstico; además, su principal asociación a nivel mundial se encuentra en del área ocupacional (5); los granjeros son los más afectados con 1,3\%-12,9\% $(8,9)$, seguidos de los cuidadores de palomas con $8 \%-10,4 \%(10,11)$, así como las personas con exposiciones no laborales tales como el tabaco con 5,2\% (12), nadadores de piscinas con $27 \%$ (13) y personas expuestas a aires acondicionados contaminados con $15 \%$ (14), algo muy común en el medio. La no asociación del antígeno químico ácido oxálico como un agente causante de la enfermedad en la literatura mundial motiva la publicación académica.

\section{Presentación del caso}

Es una paciente femenina de 35 años, con cuadro clínico de 5 años, caracterizado por tos seca persistente, ocasionalmente con expectoración hialina, agravada por factores ambientales (polvo), asociada con escala modificada de disnea (mMRC) 3. Se manejó inicialmente por alergología como bronquitis alérgica: anticolinérgicos, corticosteroides inhalados, agonistas $\beta_{2} \mathrm{y}$ antihistamínicos sin respuesta. Tenía un antecedente de exposición ambiental a ácido oxálico cada 8 días por 3 años con el cual lavaba el baño sin la debida protección hasta hace 5 años. En el examen físico en su primera consulta se encontraron signos vitales que constan de pulsioximetría $95 \%$ sobre el nivel del mar, frecuencia cardíaca (FC) de 65 latidos por minuto (lpm), frecuencia respiratoria (FR) de 16 respiraciones por minuto (rpm), presión arterial (PA) de 100/60 mm Hg, peso de $64 \mathrm{~kg}$ y una talla de 1,52 m para un índice de masa corporal (IMC) de 27,7, y auscultación pulmonar con crépitos espiratorios en los dos tercios inferiores de ambos campos pulmonares.

Con lo anterior se procedió a realizar pruebas de función pulmonar, en las que se encontraron espirometría de volumen espiratorio forzado el primer segundo/capacidad vital forzada $\left(\mathrm{VEF}_{1} / \mathrm{CVF}\right): 88$, con $\mathrm{CVF}$ predicha para la edad y sexo 3,18 , con pre- $\beta_{2} 1,77$ y pos- $\beta_{2} 1,78$, lo cual sugiere una restricción moderadamente grave no reversible; volúmenes pulmonares con capacidad pulmonar total (TLC) predicha para la edad y sexo 4,43 , con pre- $\beta_{2} 2,95$ y pos- $\beta_{2} 2,92$, lo cual sugiere una restricción moderada sin atrapamiento de aire con resistencias conservadas y capacidad de difu- 
sión pulmonar (DLCO) teórica de 18,66 (en este caso, 10,27) para una disminución moderada. Se obtuvo una tomografía axial computarizada de alta resolución (TACAR) de tórax (Figura 1) con múltiples nódulos centrolobulillares, engrosamiento peribroncovascular, opacidades reticulares y vidrio esmerilado subpleural y bibasal compatibles con NH crónica frente a neumonía intersticial usual (NIU), para lo cual se indicó una biopsia con videotoracoscopia (Figura 2) en la que, debido a los granulomas e infiltrado de predominio linfocitico, se sospechó NH.

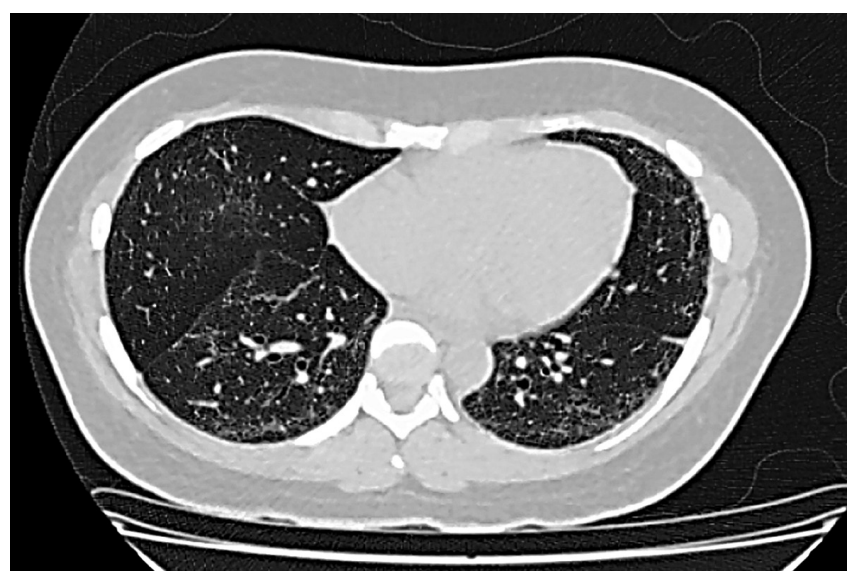

Figura 1. TACAR de tórax, nódulo centrolobulillar, engrosamiento peribroncovascular, opacidades reticulares y vidrio esmerilado subpleural y bibasal sugestivo de NH crónica o NIU.

Se inició el manejo con prednisolona a $1 \mathrm{mg} / \mathrm{kg} /$ día por un mes; posteriormente, se inició el desescalonamiento a 6 meses; al final del tratamiento, la paciente refirió empeoramiento del cuadro basal con aparición de episodios de hipertensión, depresión, estrías y episodios de hiperglucemia atribuidos al uso de corticosteroides, por lo cual se suspendieron. Ante la presencia de fibrosis se optó en junta médica por neumología el inicio de pirfenidona frente a rituximab, pero hubo una negativa por parte de la paciente ante sus fuertes deseos de gestación, por lo cual el servicio de neumología indicó el trasplante pulmonar con el fin de mejorar la supervivencia. Actualmente se encuentra en lista de espera para el trasplante ortotópico.

\section{Discusión}

En el anterior caso, la paciente se había expuesto al ácido oxálico, un producto químico que hace parte de los ácidos dicarboxílicos y el cual se encuentra ampliamente distribuido en productos de aseo, cuya comercialización no se encuentra regulada, a pesar de que la relación de $\mathrm{NH}$ y el grupo químico está plenamente identificada; sin embargo, los casos reportados de este agente especifico (ácido oxálico) como causante de la enfermedad son nulos, lo cual motiva el reporte del caso. Actualmente existen criterios diagnósticos, pero ninguno ha sido validado (15); se ha propuesto para las formas crónicas los criterios diagnósticos expresados en la Tabla 1; los cambios sugestivos en la biopsia pulmonar comprenden fibrosis pulmonar variable, destrucción de la arquitectura pulmonar, e infiltración linfocítica y mononuclear con granulomas asociados.

Tabla 1. Criterios diagnósticos de $\mathrm{NH}$

Evidencia de exposición y anticuerpos específicos contra el antígeno

Comportamiento clínico de una enfermedad pulmonar intersticial

Linfocitosis en el lavado bronquioalveolar

Opacidades de vidrio esmerilado, nódulos centrolobulillares mal definidos y atenuación de mosaico en imágenes inspiratorias y de atrapamiento de aire en imágenes de TAC espiratorias

Patrón restrictivo en espirometría asociado con hipoxemia y DLCO disminuido

Biopsia de pulmón con cambios sugestivos

Tomado de: Selman M et al. Semin Respir Crit Care Med. 2012;33(05):543-54.

A la paciente estudiada de forma tardía se le identificaron 5 criterios de los 6 antes descritos, por lo que se contextualiza como una enfermedad pulmonar de hipersensibilidad. El retiro del antígeno agresor no configura cambios de mejoría y la enfermedad evoluciona a más de 6 meses, lo que constituye por definición una enfermedad crónica; se inició la segunda línea con corticosteroides sistémicos a dosis moderada con evolución tórpida, una forma fenotípica poco común, ya que la mayoría de pacientes responde a la inmunosupresión (16), por lo que se optó por terapia de cuarta línea con 

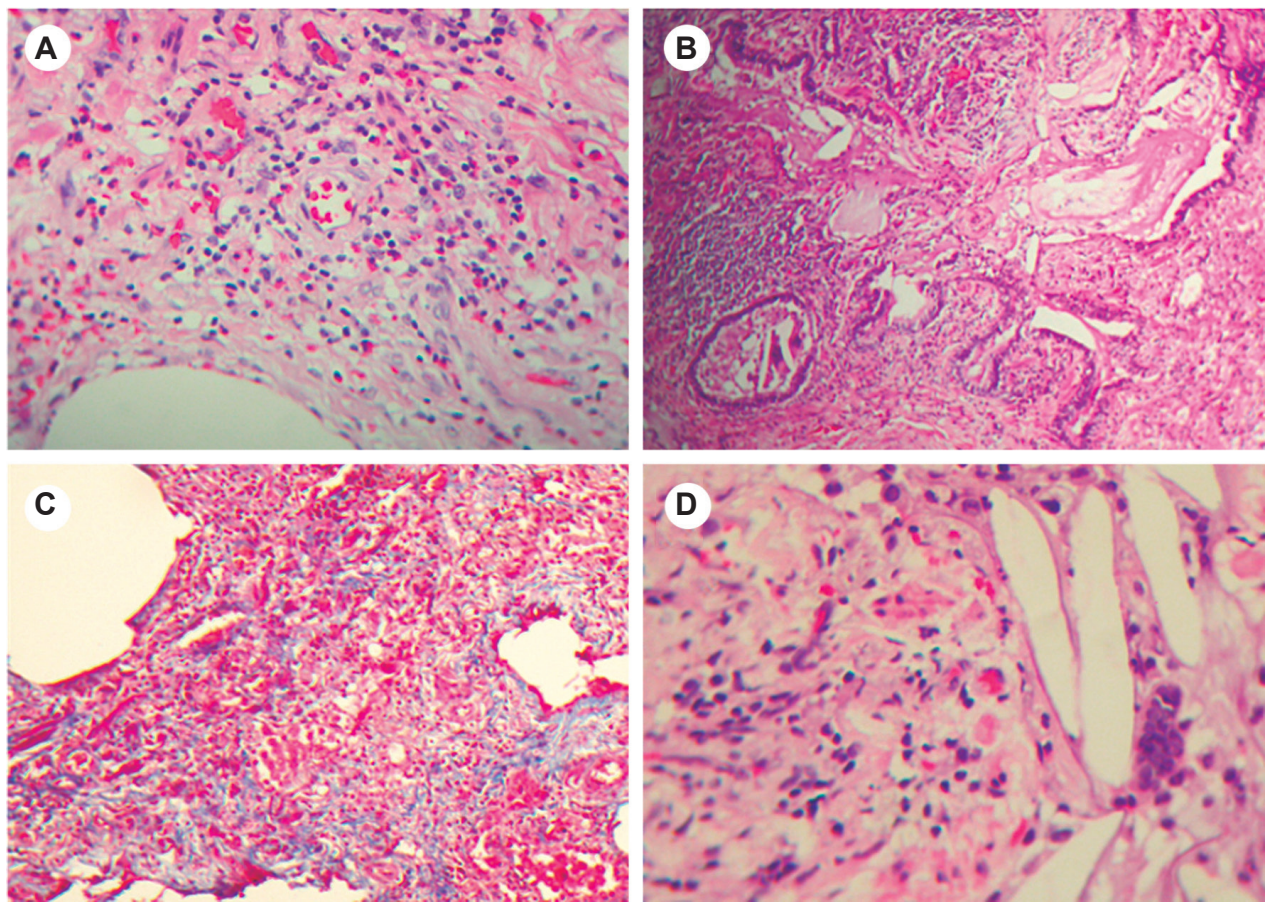

Figura 2. Coloración hematoxilina-eosina de la biopsia pulmonar. A. $40 \mathrm{X}$, infiltrado intersticial a base de linfocitos, histiocitos, plasmocitos y eosinófilos. B. 10 $\mathrm{X}$, compromiso bronquiolar. $\mathrm{C}$. $10 \mathrm{X}$, fibrosis intersticial en parches. D. $100 \mathrm{X}$, granuloma tipo colesterol.

trasplante pulmonar ante la negativa de la paciente a otras terapias; esta opción terapéutica posee una tasa de recurrencia de solo $6 \%$ (17) y una tasa de supervivencia a 5 años del $89 \%$ (17), por lo que constituye la terapia idónea para impactar en mortalidad cuando se han agotado otros recursos.

\section{Conclusiones}

La NH representa una reacción inmunológica a un agente inhalado, con una prevalencia muy baja que justifica su infradiagnóstico. Una amplia gama de ocupaciones con contacto de antígenos orgánicos e inorgánicos aumenta el riesgo de desarrollar NH; el diagnóstico precoz es importante, por la reversibilidad inicial. En el caso descrito, el diagnóstico se realiza de forma tardía, lo que impacta en la cronicidad y evolución de los síntomas, con poca respuesta a las terapias iniciales, por lo que se indica una terapia con trasplante pulmonar, el cual posee una alta tasa de éxito con respecto a mortalidad y recurrencia.

\section{Referencias}

1. Spagnolo P, Rossi G, Cavazza A, Bonifazi M, Paladini I, Bonella F, et al. Hypersensitivity Pneumonitis: A Comprehensive Review. J Investig Allergol Clin Immunol. 2015;25(4):237-50.

2. Costabel U, Bonella F, Guzman J. Chronic Hypersensitivity Pneumonitis. Clin Chest Med. 2012;33(1):151-63.

3. Vasakova M, Morell F, Walsh S, Leslie K, Raghu G. Hypersensitivity Pneumonitis: Perspectives in Diagnosis and Management. Am J Respir Crit Care Med. 2017;196(6):680-9.

4. Pepys J, Riddell RW, Citron KM, Clayton YM. Precipitins Against Extracts of Hay and Moulds in the Serum of Patients with Farmer's Lung, Aspergillosis, Asthma, and Sarcoidosis. Thorax. 1962;17(4):366.

5. Quirce S, Vandenplas O, Campo P, Cruz MJ, de Blay F, Koschel D, et al. Occupational hypersensitivity pneumonitis: an EAACI position paper. Allergy. 2016;71(6):765-79.

6. Solaymani-Dodaran M, West J, Smith C, Hubbard R. Extrinsic allergic alveolitis: incidence and mortality in the general population. QJM. 2007;100(4):233-7.

7. Thomeer MJ, Costabe U, Rizzato G, Poletti V, Demedts M. Comparison of registries of interstitial lung diseases in three European countries. Eur Respir J Suppl. 2001;32:114s-118s. 
8. Stanford CF, Hall G, Chivers A, Martin B, Nicholls DP, Evans J. Farmer's lung in Northern Ireland. Br J Ind Med. 1990;47(5):314-6.

9. Depierre A, Dalphin JC, Pernet D, Dubiez A, Faucompré C, Breton JL. Epidemiological study of farmer's lung in five districts of the French Doubs province. Thorax. 1988;43(6):429-35.

10. Banham SW, Mcsharry C, Lynch PP, Boyd G. Relationships between avian exposure, humoral immune response, and pigeon breeders' disease among Scottish pigeon fanciers. Thorax. 1986;41:274-8.

11. Rodríguez de Castro F, Carrillo T, Castillo R, Blanco C, Díaz F, Cuevas M. Relationships Between Characteristics of Exposure to Pigeon Antigens. Chest. 1993;103(4):1059-63.

12. Huuskonen MS, Husman K, Jarvisalo J, Kotimaa M, Kuusela T, Nordman H, et al. Extrinsic allergic alveolitis in the tobacco industry. Br J Ind Med. 1984;41:77-83.
13. Rose CS, Martyny JW, Newman LS, Milton DK, King TE, Beebe JL, et al. "Lifeguard lung": endemic granulomatous pneumonitis in an indoor swimming pool. Am J Public Health. 1998;88(12):1795-800.

14. Banaszak EF, Thiede WH, Fink JN. Hypersensitivity Pneumonitis Due to Contamination of an Air Conditioner. N Engl J Med. 1970;283(6):271-6.

15. Schuyler M, Cormier Y. The diagnosis of hypersensitivity pneumonitis. Chest. 1997;111(3):534-6.

16. Selman M, Buendía-Roldán I. Immunopathology, Diagnosis, and Management of Hypersensitivity Pneumonitis. Semin Respir Crit Care Med. 2012;33(05):543-54.

17. Kern RM, Singer JP, Koth L, Mooney J, Golden J, Hays S, et al. Lung transplantation for hypersensitivity pneumonitis. Chest. 2015;147(6):1558-65. 